\title{
Complex Modulus of Crosslinked High Polymers
}

\author{
Tohru MORITA ${ }^{1 *}$ and Yoshihiro SATOH ${ }^{2}$ \\ ${ }^{1}$ Nakayama-Yoshinari 3-4-34, Aoba-ku, Sendai 989-3203, Japan \\ ${ }^{2}$ Department of Mechanical Engineering, Sophia University, Chiyoda-ku, Tokyo 102-8554, Japan
}

Received June 18, 2003; final version accepted March 19, 2004

\begin{abstract}
For crosslinked high polymers, the effect of crosslinkages to the frequency-dependent complex modulus is estimated with the aid of a network model. It is then shown that it can be used to explain the deviation of experimental values for a material of crosslinked high polymers, from the theoretical values for a dilute solution of high polymers. Some discussions are given also on the relaxation modulus.
\end{abstract}

KEYWORDS: crosslinked high polymers, complex modulus, network model

\section{Introduction}

Bagley and Torvik studied the frequency-dependent complex modulus of viscoelastic materials [1]. They showed that it takes the form

$$
E^{*}(\omega)=E_{0}+E_{1}(\mathrm{i} \omega)^{\alpha}
$$

for a number of viscoelastic materials, where $E_{0}$ and $E_{1}$ are constants and $\alpha \simeq 0.5$. Those authors then argued that the term $E_{1}(\mathrm{i} \omega)^{\alpha}$ can be explained with the aid of Rouse's theory, which is a theory for a dilute solution of high polymers; see [12].

In the present paper, we study the complex shear modulus of a material of crosslinked high polymers. We first observe that the rule (1) applies fairly well at high frequencies for the material, and find some deviation at low frequencies.

For crosslinked high polymers, the network model is a typical model, which takes account of the effects of entanglement of polymer molecules and crosslinking; see [3], [11]. We now study the contribution of networks in a network model to the shear modulus, and show that it can be used to explain the above-mentioned deviation of shear modulus of a real material at low frequencies.

In Section 2, a compact derivation of the term $E_{1}(\mathrm{i} \omega)^{\alpha}$ with $\alpha=0.5$ in the rule (1) is given on the basis of the Rouse theory. In Section 3, the contribution of networks in the network model to the shear modulus is obtained with the aid of the Rouse theory. In Section 4, an experimental result is compared with the theoretical result. We present a simplified expression to be compared with experiments in Section 5. In Section 6, concluding remarks are given on the present analysis of the complex modulus. The implication of the present analysis to the relaxation modulus and its inverse Laplace transform is stated in Section 7. This provides another viewpoint to the analysis given in the preceding sections.

\section{Rouse, Bagley and Torvik Theory}

In the Rouse theory of dilute solution of polymers, a polymer molecule is regarded to consist of submolecules, and the mode of the motion of a polymer molecule is labeled by an integer $p=1,2, \ldots, N$, where $N$ is the total number of the submolecules in the polymer molecule. For the mode $p$, the relaxation time $\tau_{p}$ is estimated to be

$$
\frac{1}{\tau_{p}}=C_{1} \lambda_{p}=4 C_{1} \sin ^{2}\left(\frac{p \pi}{2(N+1)}\right),
$$

where $C_{1}=6 B k_{\mathrm{B}} T / \sigma^{2}$. Here $B$ is the mobility of a submolecule, $k_{\mathrm{B}}$ is the Boltzmann constant, $T$ is the absolute temperature, and $\sigma^{2}$ is the mean-square separation of the ends of a submolecule. The complex shear modulus $G^{*}(\omega)$ of the system at frequency $\omega$ was expressed as

$$
G^{*}(\omega)=\mathrm{i} \omega \eta^{*}=\mathrm{i} \omega\left(\eta_{\mathrm{s}}+n k_{\mathrm{B}} T \sum_{p=1}^{N} \frac{1}{\frac{1}{\tau_{p}}+\mathrm{i} \omega}\right) .
$$

Here $\eta_{\mathrm{s}}$ is the viscosity of the solvent, and $n$ is the total number of polymer molecules in unit volume.

* Corresponding author. E-mail: senmm@hkg.odn.ne.jp 
When $N$ is so large that $\pi /[2(N+1)]=1 /\left[2 \sqrt{C_{1} \tau_{1}}\right] \ll 1$ and $\omega \gg 1 / \tau_{1}$, the sum on the righthand side of (3) is evaluated as

$$
\begin{aligned}
\sum_{p=1}^{N} \frac{1}{\frac{1}{\tau_{p}}+\mathrm{i} \omega} & =\frac{\sqrt{\tau_{1}}}{2 \sqrt{C_{1}}} \int_{0}^{\pi / 2} \frac{1}{\mathrm{i} \omega+4 C_{1} \sin ^{2} \theta} \mathrm{d} \theta \\
& =\frac{\sqrt{\tau_{1}}}{8 \sqrt{C_{1}} \sqrt{\mathrm{i} \omega}} \int_{0}^{2 \pi} \frac{1}{\sqrt{\mathrm{i} \omega}-2 \mathrm{i} \sqrt{C_{1}} \sin \theta} \mathrm{d} \theta=\frac{\pi \sqrt{\tau_{1}}}{8 C_{1} \sqrt{\mathrm{i} \omega}} \frac{1}{\left(1+\mathrm{i} \frac{\omega}{4 C_{1}}\right)^{1 / 2}}
\end{aligned}
$$

When $\omega /\left(4 C_{1}\right)=\omega \tau_{1} \pi^{2} /\left[4(N+1)^{2}\right] \ll 1$, we obtain

$$
G^{*}(\omega) \simeq \mathrm{i} \omega \eta_{\mathrm{s}}+n k_{\mathrm{B}} T \frac{\pi}{8 C_{1}} \tau_{1}^{1 / 2}(\mathrm{i} \omega)^{1 / 2} .
$$

This expression was derived by Bagley and Torvik [1], by using approximation $\tau_{p}=\tau_{1} / p^{2}$ for small $p$, for the range of frequency $25<\omega \tau_{1} \ll N^{2}$.

\section{System of Square Networks}

In (2), $\lambda_{p}=4 \sin ^{2}(p \pi /[2(N+1)])=2-2 \cos (p \pi /[N+1])$ for $p=1,2, \ldots, N$. They are the eigenvalues of the equation for a linear chain

$$
2 u(i)-u(i-1)-u(i+1)=\lambda u(i), \quad i=1,2, \ldots, N,
$$

where $u(0)=u(N+1)=0$.

We now consider the correlated motion of adjacent parts of two polymer molecules or of different parts of a single polymer molecule, when the two parts come near with each other by entanglement or by connection to a common crosslinking point. We now call those connected parts as a whole crosslinkages. In order to estimate the effect of such crosslinkages to the shear modulus, we consider a system of networks which are square lattices of $N_{2} \times N_{2}$ lattice sites, where a crosslinkage is situated at every lattice site; see [3], [11]. Assuming the same coupling constants between nearest neighbour lattice sites, the equation corresponding (6) for a network is

$$
\begin{array}{r}
4 u(i, j)-u(i-1, j)-u(i+1, j)-u(i, j-1)-u(i, j+1)=\lambda u(i, j), \\
i=1,2, \ldots, N_{2} ; j=1,2, \ldots, N_{2},
\end{array}
$$

where $u(0, j)=u(i, 0)=u\left(N_{2}+1, j\right)=u\left(i, N_{2}+1\right)=0$ for $i=1,2, \ldots, N_{2}$ and $j=1,2, \ldots, N_{2}$. Now the eigenvalues are

$$
\lambda_{p, q}=4-2 \cos \frac{p \pi}{N_{2}+1}-2 \cos \frac{q \pi}{N_{2}+1},
$$

for $p=1,2, \ldots, N_{2}$ and $q=1,2, \ldots, N_{2}$, and the relaxation time $\tau_{p, q}$ for the mode $p, q$ is given by $1 / \tau_{p, q}=C_{2} \lambda_{p, q}$. Here $C_{2}$ is the quantity for a crosslinkage, corresponding to $C_{1}$ for a submolecule.

When $N_{2}$ is sufficiently large, the sum on the righthand side of (3) is now replaced by

$$
\begin{aligned}
\sum_{p=1}^{N_{2}} \sum_{q=1}^{N_{2}} \frac{1}{\frac{1}{\tau_{p, q}}+\mathrm{i} \omega} & =2 C_{2} \tau_{1,1} \int_{0}^{\pi} \int_{0}^{\pi} \frac{1}{\mathrm{i} \omega+4 C_{2}-2 C_{2} \cos \theta_{1}-2 C_{2} \cos \theta_{2}} \mathrm{~d} \theta_{1} \mathrm{~d} \theta_{2} \\
& =2 C_{2} \tau_{1,1} \pi^{2} \frac{2}{\pi\left(\mathrm{i} \omega+4 C_{2}\right)} K\left(\frac{4 C_{2}}{\mathrm{i} \omega+4 C_{2}}\right) .
\end{aligned}
$$

Here $2 C_{2} \tau_{1,1}=\left(N_{2}+1\right)^{2} / \pi^{2}$, and $K(k)$ is the complete elliptic integral of the first kind. The last equality is known in the theory of the lattice Green's function on the square lattice; see [6]. The numerical procedure of calculating the complete elliptic integrals of the first and second kinds, $K(k)$ and $E(k)$, for complex modulus $k$, is given in [7]; see also [10], $[8,9]$.

If we assume that there are $n_{2}$ networks in unit volume of the system, the contribution due to them is estimated by replacing $n$ and the sum in (3) by $n_{2}$ and the sum given by (9). By adding this contribution to the one (5), we obtain

$$
G^{*}(\omega) \simeq b \omega^{1 / 2}+\mathrm{i} b \omega^{1 / 2}+c M_{1}\left(\omega / \omega_{1}\right),
$$

where $b=n k_{\mathrm{B}} T \pi \tau_{1}^{1 / 2} /\left(8 C_{1} \sqrt{2}\right), c=2 n_{2} k_{\mathrm{B}} T C_{2} \tau_{1,1} \pi^{2}, \omega_{1}=4 C_{2}$, and

$$
M_{1}(x)=\frac{\mathrm{i} x}{\mathrm{i} x+1} \frac{2}{\pi} K\left(\frac{1}{\mathrm{i} x+1}\right) .
$$




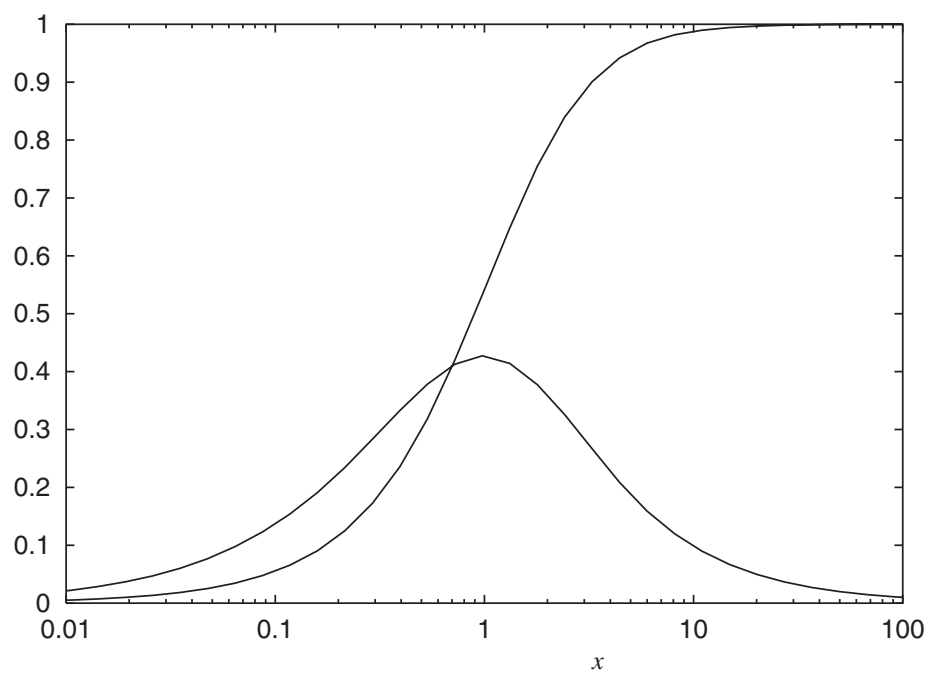

Fig. 1. $\quad M_{1}(x)=\frac{\mathrm{i} x}{\mathrm{i} x+1} \frac{2}{\pi} K\left(\frac{1}{\mathrm{i} x+1}\right)$ as a function of $x$. The upper and lower curves are the real and imaginary parts, respectively.

Here we put $\eta_{\mathrm{s}}=0$ since we have no solvent. The real and imaginary parts of the function $M_{1}(x)$ are plotted as a function of $x$ in Fig. 1. From this figure, we see that the value $\omega_{1}$ in $M_{1}\left(\omega / \omega_{1}\right)$ is the characteristic value in the sense that the imaginary part has a peak at $\omega=\omega_{1}$ and the real part is nearly equal to 0 and 1 when $\omega<0.1 \omega_{1}$ and $\omega>10 \omega_{1}$, respectively.

In this place, we try to include the effect of randomness in crosslinkages. The distance between neighbouring crosslinkages along a polymer molecule may be very small or may be larger by factor 10 or more. When the distance is larger, the coupling between neighbouring crosslinkages will be weaker and the frequency $\omega_{1}$ will be reduced, and then the effective mass of a crosslinkage will be larger and the frequency $\omega_{1}$ will be reduced further. As the result, we expect a wide distribution of the value of $\omega_{1}$. In order to include the effect of this distribution of the value of $\omega_{1}$, we replace the term $c M_{1}\left(\omega / \omega_{1}\right)$ in $(10)$ by an average, so that

$$
G^{*}(\omega) \simeq b \omega^{1 / 2}+\mathrm{i} b \omega^{1 / 2}+c \int p\left(\omega_{1}\right) M_{1}\left(\frac{\omega}{\omega_{1}}\right) \mathrm{d} \omega_{1},
$$

where $p\left(\omega_{1}\right)$ is the probability distribution function of $\omega_{1}$. In particular, we define $M_{L}(x)$ for integer $L$, by

$$
M_{L}(x)=\frac{1}{L} \sum_{n=0}^{L-1} M_{1}\left(2^{n} x\right),
$$

and we adopt

$$
G^{*}(\omega) \simeq b \omega^{1 / 2}+\mathrm{i} b \omega^{1 / 2}+c M_{L}\left(\omega / \omega_{0}\right),
$$

in place of (10) or (12), in the following section. The curves of the real and imaginary parts of the average $M_{L}(x)$ for $L=24$ are shown in Fig. 2 .

\section{Comparison with Experiment}

We now study the complex shear modulus of a vulcanized acrylic rubber, that is acrylic rubber Nipol AR54 vulcanized with $0.74 \%$ ammonium benzoate. The result of the experiment is shown in Fig. 3. If (5) given by Rouse's theory applies with $\eta_{\mathrm{s}}=0$, the real and imaginary parts of $G^{*}(\omega), G^{\prime}(\omega)$ and $G^{\prime \prime}(\omega)$, are given by $G^{\prime}(\omega)=G^{\prime \prime}(\omega)=$ $b \omega^{1 / 2}$ with a constant $b$. We introduce a constant term in the real part $G^{\prime}(\omega)$ following (1), and fitted the real and imaginary parts to $G^{\prime}(\omega)=a+b \omega^{1 / 2}$ and $G^{\prime \prime}(\omega)=b \omega^{1 / 2}$ in frequency range $2 \leqq \omega \leqq 100$. Then we obtain

$$
\begin{gathered}
G^{*}(\omega)=a+b \omega^{1 / 2}+\mathrm{i} b \omega^{1 / 2}, \\
a=0.127020, \quad b=0.016821 .
\end{gathered}
$$

The real and imaginary parts of this function are shown by dashed lines in Fig. 3. This shows that the motion of many of high polymers is well described by the motion of a chain in frequencies $2 \leqq \omega \leqq 100$.

In Fig. 4(a), the deviations of $G^{\prime}(\omega)$ and $G^{\prime \prime}(\omega)$ from their high-frequency asymptotes given by (14) with (15) are shown. In the same figure, the curves of $0.01 \pi\left[M_{1}(\omega / 0.1)-1\right]$ as a function of $\omega$ are shown. From this figure, we can say that the agreement is surprizingly good, considering that the model we adopt is a very crude one.

In Fig. 4(b), the deviations of $G^{\prime}(\omega)$ and $G^{\prime \prime}(\omega)$ from their high-frequency asymptotes given by (14) are compared with the curves of $0.127\left[M_{24}(\omega / 0.5)-1\right]$. Now the agreement is very much improved. 


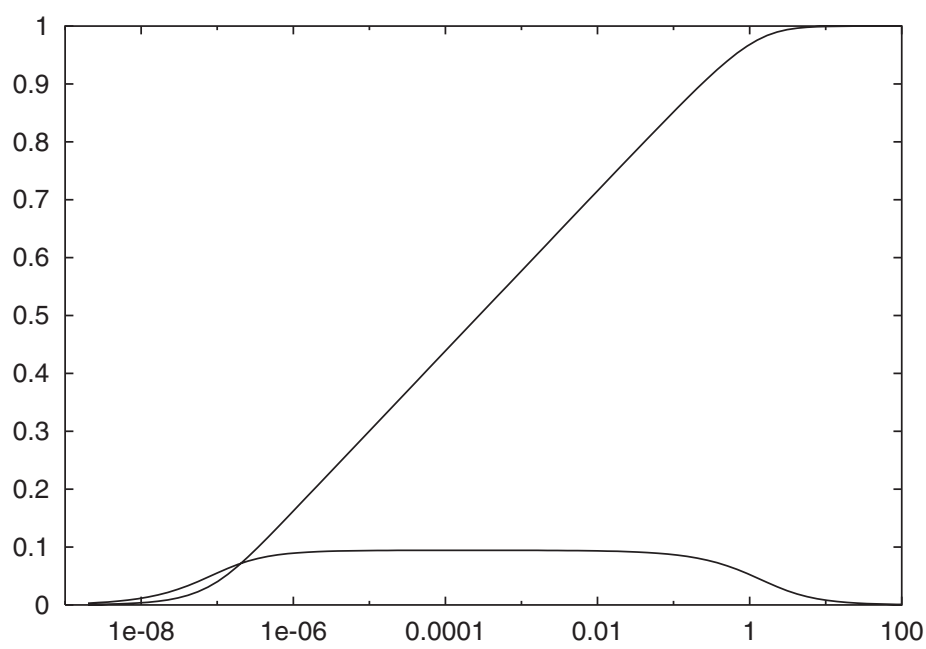

Fig. 2. $\quad M_{24}(x)=\frac{1}{24} \sum_{n=0}^{23} M_{1}\left(2^{n} x\right)$ as a function of $x$, where $M_{1}(x)=\frac{\mathrm{i} x}{\mathrm{i} x+1} \frac{2}{\pi} K\left(\frac{1}{\mathrm{i} x+1}\right)$. The upper and lower curves are the real and imaginary parts, respectively.

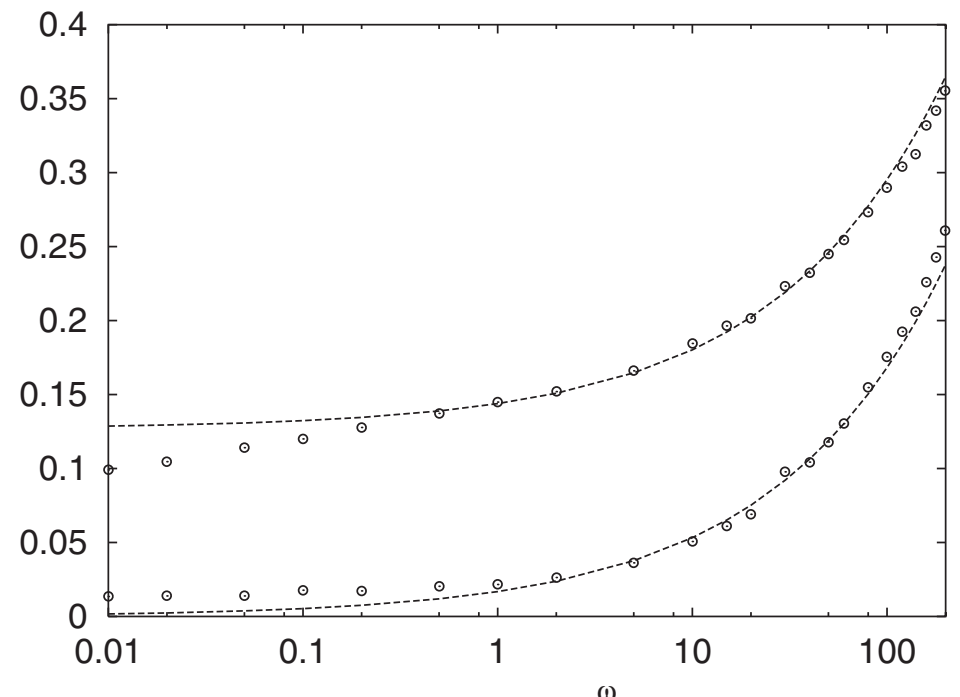

Fig. 3. Experimental result of $G^{\prime}(\omega)$ and $G^{\prime \prime}(\omega)$ shown by circles, and the asymptotes at high frequencies given by (14) with (15), shown in dashed lines. The upper and lower values are the real and imaginary parts, respectively.
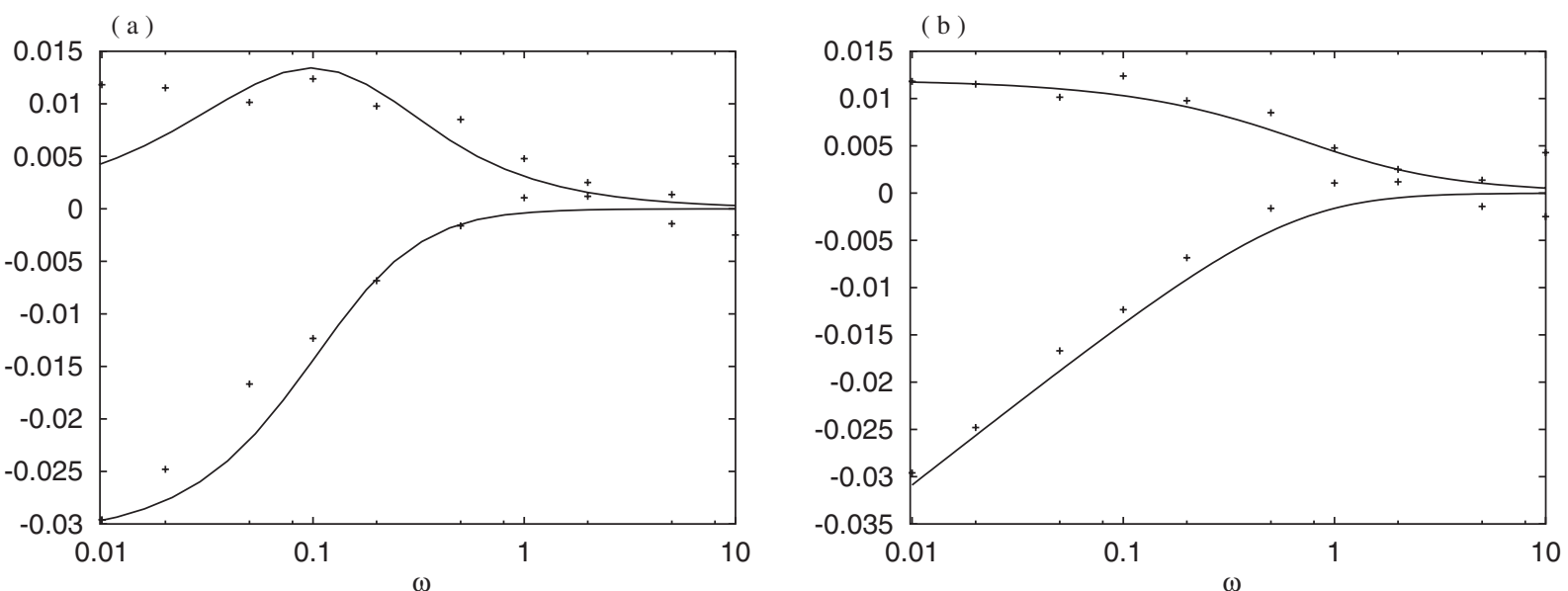

Fig. 4. The deviations of experimental values of $G^{\prime}(\omega)$ and $G^{\prime \prime}(\omega)$ from their high frequency asymptotes (14) with (15), shown by crosses, are compared with the curves for the real and imaginary parts of $0.1 \pi\left[M_{1}(\omega / 0.1)-1\right]$ in $(\mathrm{a})$, and with the curves for the real and imaginary parts of $0.127\left[M_{24}(\omega / 0.5)-1\right]$ in (b). The lower and upper values are the real and imaginary parts, respectively. 


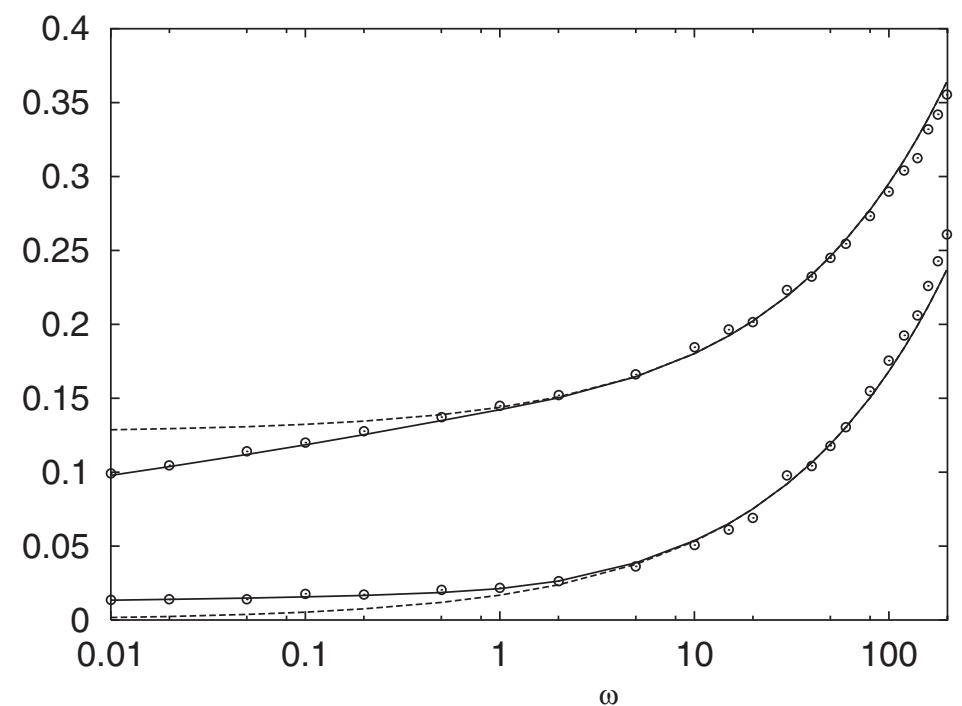

Fig. 5. Experimental result of $G^{\prime}(\omega)$ and $G^{\prime \prime}(\omega)$ shown by circles, compared with the curves, shown by solid lines, for (13) with constants given in (16). Dashed lines show the asymptotes at high frequencies given by (14) with (15). The upper and lower values are the real and imaginary parts, respectively.

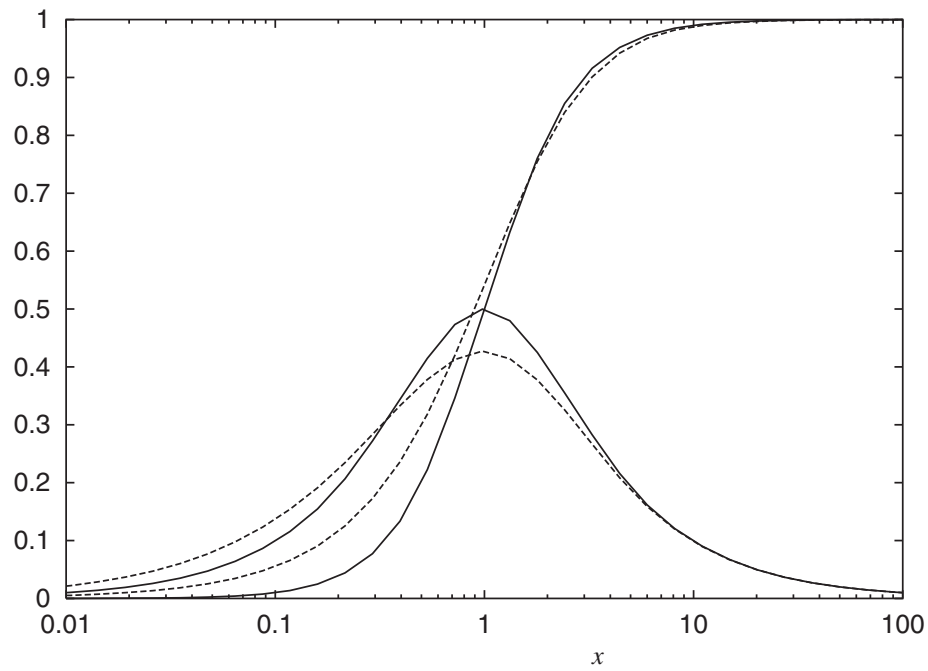

Fig. 6. $\quad M_{0}^{*}(x)=\frac{\mathrm{i} x}{1+\mathrm{ix}}$ shown by solid lines, compared with $M_{1}(x)=\frac{\mathrm{i} x}{\mathrm{i} x+1} \frac{2}{\pi} K\left(\frac{1}{\mathrm{i} x+1}\right)$ shown by dashed lines, as a function of $x$. The upper and lower curves are the real and imaginary parts, respectively.

In Fig. 5, we show the curves of (13) with the constants given by

$$
b=0.016821, \quad L=24, \quad c=0.127, \quad \omega_{0}=0.5 .
$$

The curves are compared with the experimental data and the high-frequency asymptotes given by (14) with (15). We observe a very good agreement of the curves with experimental data except at very high frequencies.

\section{Simplified Expression}

In Section 3, we discussed the contribution to the shear modulus due to a system of networks. The result is shown in Fig. 1. The curves recall us the curves of $\mathrm{i} x /(1+\mathrm{i} x)$ for the elastic modulus of Maxwell's model of viscoelasticity; see, e.g., [11]. In Fig. 6, we compare the curves of $M_{1}(x)$ and $M_{0}^{*}(x)=\mathrm{i} x /(1+\mathrm{i} x)$. Considering that the approximation to use $M_{1}(x)$ is a very crude one, we can use $M_{0}^{*}(x)$ in place of $M_{1}(x)$. Then we may use the average

$$
\begin{aligned}
M_{L}^{*}(x) & =\frac{1}{L} \int_{0}^{L} M_{0}^{*}\left(2^{n} x\right) \mathrm{d} n=\frac{1}{L \ln 2} \int_{2^{-L}}^{1} M_{0}^{*}\left(x / x_{1}\right) \frac{\mathrm{d} x_{1}}{x_{1}} \\
& =\frac{1}{L \ln 2} \log \frac{1+\mathrm{i} 2^{L} x}{1+\mathrm{i} x},
\end{aligned}
$$




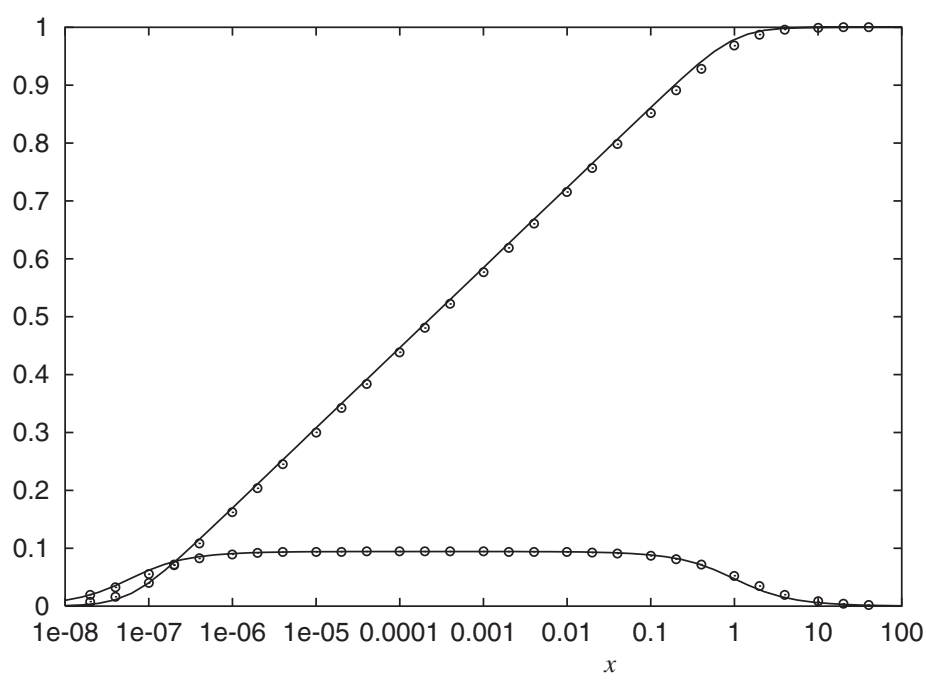

Fig. 7. $M_{24}^{*}(x)$ shown by solid lines, compared with $M_{24}(x)$ shown by circles, as a function of $x$. The upper and lower curves are the real and imaginary parts, respectively.

in place of $M_{L}(x)$. Comparison is made of these functions for $L=24$ in Fig. 7. We observe that they agree very well with each other.

Asymptotic values of the function $M_{L}^{*}(x)$ are given by

$$
M_{L}^{*}(x)= \begin{cases}0, & x \ll 2^{-L}, \\ 1+\frac{1}{L \ln 2}\left[\ln x+\frac{\pi}{2} \mathrm{i}\right], & 2^{-L} \ll x \ll 1, \\ 1, & 1 \ll x .\end{cases}
$$

In the case of Fig. $7,2^{-L}=2^{-24} \simeq 0.596 \times 10^{-7}$.

Here we propose to use

$$
G^{*}(\omega) \simeq a^{*}+b \omega^{1 / 2}+\mathrm{i} b \omega^{1 / 2}+c^{*} M_{L^{*}}^{*}\left(\omega / \omega_{0}\right)
$$

in fitting experimental data. Here $b$ and $c^{*}$ are determined by the amounts of submolecules in polymers and crosslinkages, respectively, and $\omega_{0}$ and $2^{-L^{*}} \omega_{0}$ are the highest and lowest characteristic frequencies of the motion of crosslinkages, and $a^{*}$ is the elastic term.

The result (13) with (16) may well be approximated by (19) with $a^{*}=0, c^{*}=c=0.127, L^{*}=L=24$, and $b$ and $\omega_{0}$ given in (16). As far as we consider the frequencies satisfying $\omega>0.01$, we may choose any values of $L^{*}, a^{*}$ and $c^{*}$ in so far as $2^{-L^{*}+1}<0.01 / \omega_{0}, c^{*} / L^{*}=c / L=0.127 / 24$ and $a^{*}+c^{*}=c=0.127$ are satisfied. For instance, we may choose $L^{*}=10, c^{*}=10 \times 0.127 / 24=0.05292$ and $a^{*}=0.127-0.05292=0.07408$, and $b$ and $\omega_{0}$ given in (16).

We now study the complex shear modulus of another sample of vulcanized acrylic rubber, that is acrylic rubber Nipol AR54 vulcanized with $2.88 \%$ ammonium benzoate. The result of the experiment is shown in Fig. 8. We fitted the data to (14) in frequency range $2 \leqq \omega \leqq 100$. Then we obtained

$$
a=0.133475, \quad b=0.014398 .
$$

The curves of this function are shown in Fig. 8. No appreciable deviation is observed in the imaginary part. This means that $\omega_{0}$ is less than 0.01 . The agreement in the real part is not so good as in the preceding data. In the present sample, we have more crosslinkages and the data is far from a system which can be regarded to be one of isolated polymer molecules with some corrections. We suppose that this is the reason why an overall agreement is not achieved in this case.

When we compare the values of $a$ and $b$ in (15) for the previous sample and (20) for the present sample, we observe that the value of $a$ in (20) is greater than the value in (15) and the value of $b$ in (20) is less than the value in (15). These correspond to the facts that the number of crosslinkages is increased and the number of submolecules participating in the motions with higher frequencies is reduced in the present sample.

\section{Concluding Remarks on Complex Shear Modulus}

We studied the frequency dependence of the complex shear modulus of a material of crosslinked high polymers. For the experimental result shown in Fig. 3, the main contribution is attributed to isolated high polymer molecules for all frequencies. At low frequencies, a systematic deviation is observed. We regard this as the effect of entanglement of 


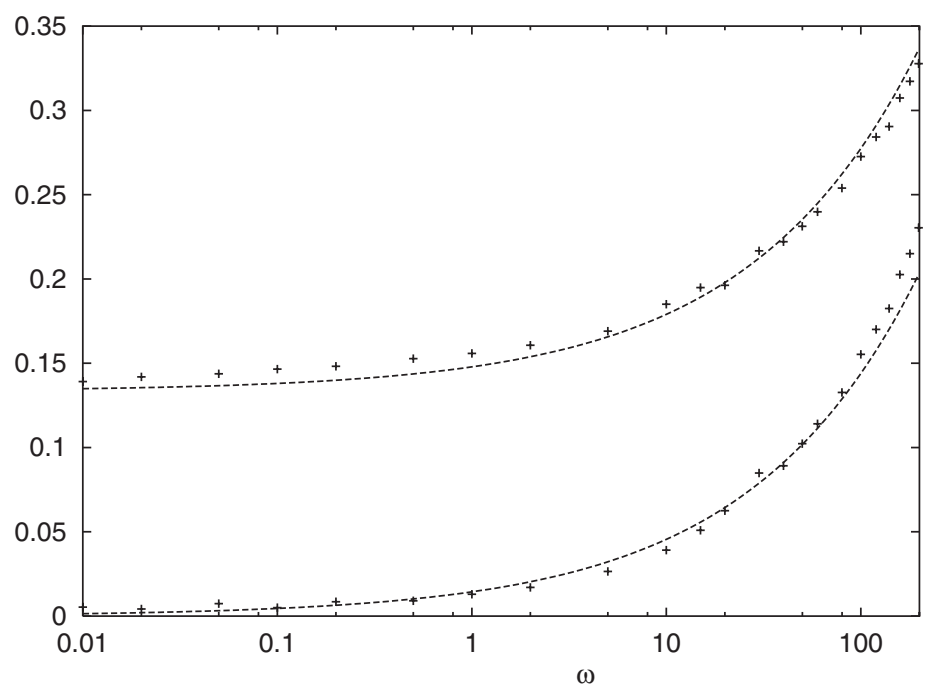

Fig. 8. Experimental result of $G^{\prime}(\omega)$ and $G^{\prime \prime}(\omega)$ for the second sample shown by crosses, and the asymptotes at high frequencies given by (14) with (20), shown in dashed lines. The upper and lower values are the real and imaginary parts, respectively.

polymer molecules and crosslinking. It is taken into account in the present paper in the form of networks of finite square lattice where the lattice sites represent crosslinkages. The coupling between the crosslinkages is weak, and its effect is appreciable only at low frequencies. Considering the distribution of the distance between neighbouring crosslinkages, an average was taken of the contribution from networks, and as the result, we could attain a very good agreement.

In Section 5, we proposed to use (19) in fitting experimental data. (17) shows that $M_{L}^{*}\left(\omega / \omega_{0}\right)$ is the average of $M_{0}^{*}\left(\omega / \omega_{1}\right)=\mathrm{i} \omega /\left(\omega_{1}+\mathrm{i} \omega\right)$ with the probability distribution given by

$$
p\left(\omega_{1}\right)= \begin{cases}\frac{1}{L \ln 2} \frac{1}{\omega_{1}}, & 2^{-L} \omega_{0}<\omega_{1}<\omega_{0}, \\ 0, & \text { otherwise. }\end{cases}
$$

If a network of lattice constant $r_{1}$ has the contribution $M_{1}^{*}\left(\omega / \omega_{1}\right)$ with $\omega_{1}=\beta r_{1}^{\gamma}$, this probability distribution (21) of $\omega_{1}$ corresponds to the probability distribution of $r_{1}$, in the form:

$$
p_{r}\left(r_{1}\right)= \begin{cases}\frac{C_{r}}{r_{1}}, & r_{0}<r_{1}<r_{0}^{\prime} \\ 0, & \text { otherwise }\end{cases}
$$

where

$$
\omega_{0}=\beta r_{0}^{\gamma} \quad \text { and } \quad 2^{-L} \omega_{0}=\beta r_{0}^{\prime \gamma}, \quad \text { and } \quad C_{r}^{-1}=\int_{r_{0}}^{r_{0}^{\prime}} \frac{1}{r_{1}} \mathrm{~d} r_{1}=\ln \left(r_{0}^{\prime} / r_{0}\right) .
$$

In the preceding section, the contribution $M_{1}\left(\omega / \omega_{1}\right)$ is replaced by $M_{0}^{*}\left(\omega / \omega_{1}\right)$. This means that a network is replaced by a number of Maxwell models of the same frequency $\omega_{1}$. The Maxwell model may be regarded to represent a pair of crosslinkages connected by a polymer molecule. Then the characteristic frequency $\omega_{1}$ is the $1 / \tau_{1}$ or $1 / \tau_{2}$ for the Rouse model of $N=2$, and hence $4 C_{3} \sin ^{2}(p \pi / 6)$ for $p=1,2$. Now $1 / \tau_{1}=C_{3}$ and $1 / \tau_{2}=3 C_{3}$. Here $C_{3}$ represents $C_{1}=$ $6 B k_{\mathrm{B}} T / \sigma^{2}$ in Section 2. $\sigma^{2}$ in the present case is the length of the part of the polymer molecule, connecting two crosslinkages. We can justify (21) if we can show that the probability distribution function of the $\sigma$ is proportional to $1 / \sigma$ in an interval $\left(\sigma_{1}, \sigma_{2}\right)$. This problem is left to future.

The $1 / r_{1}$ dependence of $p_{r}\left(r_{1}\right)$ or the $1 / \sigma$ dependence of the probability distribution of $\sigma$ recalls the following fact. The probability that a random walk of $2 n$ steps on the infinite square lattice comes back to the starting point is given by $1 / n \pi$ when $n$ is large; see [2]. On the simple cubic lattice, the probability is of order of $1 / n^{3 / 2}$; see [2].

The data shown in Figs. 3 and 5 could be fitted with the present theoretical result. But the result was not so satisfactory for the data shown in Fig. 8, which involves more crosslinkages.

We did not discuss the deviation of the experimental data at higher frequencies at $\omega \geq 100$. In the Rouse theory, a polymer molecule is treated as a chain of submolecules and the motion in the scale less than the size of a submolecule was not considered. The deviation at the high frequencies was expected in the Rouse theory from the outset; see [12].

\section{Relaxation Modulus and its Inverse Laplace Transform}

In this section, we discuss the relaxation modulus $g(t)$ and its inverse Laplace transform $\rho(E)$, corresponding to the 
complex shear modulus $G^{*}(\omega)$ discussed in the preceding sections.

When a strain $\gamma(t)$ is given, the corresponding stress $\sigma(t)$ is expressed as

$$
\sigma(t)=\int_{-\infty}^{t} \frac{\mathrm{d} \gamma\left(t^{\prime}\right)}{\mathrm{d} t^{\prime}} g\left(t-t^{\prime}\right) \mathrm{d} t^{\prime}
$$

in terms of the relaxation modulus $g(t)$. When the strain is $\gamma(t)=\gamma_{0} \mathrm{e}^{\mathrm{i} \omega t}$ and the complex modulus is $G^{*}(\omega)$, the stress is given by $\sigma(t)=G^{*}(\omega) \gamma_{0} \mathrm{e}^{\mathrm{i} \omega t}$, so that $G^{*}(\omega)$ and the Laplace transform

$$
\hat{g}(s)=\int_{0}^{\infty} g(t) \mathrm{e}^{-s t} \mathrm{~d} t
$$

of $g(t)$ are related by $\hat{g}(s)=\frac{1}{s} G^{*}(-\mathrm{i} s)$.

We first consider the case when $G^{*}(\omega)$ is given by (3) and $\eta_{\mathrm{s}}=0$. Then $\hat{g}(s)$ is given by

$$
\hat{g}(s)=n k_{\mathrm{B}} T \sum_{p=1}^{N} \frac{1}{\frac{1}{\tau_{p}}+s} \simeq \frac{\sqrt{2} b}{s^{1 / 2}\left(1+\frac{s}{4 C_{1}}\right)^{1 / 2}},
$$

following (4), where $\sqrt{2} b=n k_{\mathrm{B}} T /\left(4 C_{1}\right)^{3 / 2}$. The inverse Laplace transform $g(t)$ is given by

$$
g(t)=n k_{\mathrm{B}} T \sum_{p=1}^{N} \mathrm{e}^{-t / \tau_{p}} \simeq \sqrt{2} b\left(4 C_{1}\right)^{3 / 2} \int_{0}^{\pi / 2} \mathrm{e}^{-4 C_{1} t \sin ^{2} \theta} \mathrm{d} \theta \simeq \frac{\sqrt{2} b}{\sqrt{\pi} t^{1 / 2}},
$$

when $4 C_{1} t \gg 1$.

When $G^{*}(\omega)$ is given by (19), $\hat{g}(s)$ is given by

$$
\hat{g}(s) \simeq \frac{a^{*}}{s}+b \frac{\sqrt{2}}{s^{1 / 2}}+c^{*} \frac{1}{L^{*} \ln 2} \int_{2^{-L^{*}} \omega_{0}}^{\omega_{0}} \frac{1}{s+\omega_{1}} \frac{\mathrm{d} \omega_{1}}{\omega_{1}} .
$$

By the Laplace inversion, we obtain

$$
\begin{aligned}
g(t) & \simeq a^{*}+b \frac{\sqrt{2}}{\sqrt{\pi} t^{1 / 2}}+c^{*} \frac{1}{L^{*} \ln 2} \int_{2^{-L^{*}} \omega_{0}}^{\omega_{0}} \omega_{1}^{-1} \mathrm{e}^{-\omega_{1} t} \mathrm{~d} \omega_{1} \\
& =a^{*}+b \frac{\sqrt{2}}{\sqrt{\pi} t^{1 / 2}}+c^{*} \frac{1}{L^{*} \ln 2}\left[E_{1}\left(2^{-L^{*}} \omega_{0} t\right)-E_{1}\left(\omega_{0} t\right)\right],
\end{aligned}
$$

where

$$
E_{1}(x)=-E i(-x)=\int_{x}^{\infty} t^{-1} \mathrm{e}^{-t} \mathrm{~d} t
$$

is the exponential integral; see [5]. The terms with coefficient $b$ in (26) and (27) are the terms calculated as in (24) and (25), respectively. When $1 \ll \omega_{0} t \ll 2^{L^{*}}$, we have

$$
\begin{aligned}
g(t) \simeq & a^{*}+b \frac{\sqrt{2}}{\sqrt{\pi} t^{1 / 2}}+c^{*}-c^{*} \frac{1}{L^{*} \ln 2}\left\{\gamma+\ln \left(\omega_{0} t\right)\right. \\
& \left.+O\left(2^{-L^{*}} \omega_{0} t\right)+\mathrm{e}^{-\omega_{0} t}\left(\omega_{0} t\right)^{-1}\left[1+O\left(\left(\omega_{0} t\right)^{-1}\right)\right]\right\},
\end{aligned}
$$

where $\gamma$ is Euler's constant. In Fig. 9, $g(t)$ given by (27) is shown. We observe the $\ln t$ behaviour at $2<t<10^{7}$.

From the derivation of $G^{*}(\omega)$ in Sections 2-5, we see that $\hat{g}(s)$ is expressed as

$$
\hat{g}(s)=\int_{0-}^{\infty} \rho(E) \frac{1}{s+E} \mathrm{~d} E,
$$

where

$$
\begin{aligned}
\rho(E)= & a^{*} \delta(E)+b \frac{\sqrt{2}}{\pi} \frac{2 \sqrt{C_{1}}}{\sqrt{E\left(4 C_{1}-E\right)}} \chi_{\left(0,4 C_{1}\right)}(E) \\
& +c^{*} \frac{1}{L^{*} \ln 2} \frac{1}{E} \chi_{\left(2^{-L *} \omega_{0}, \omega_{0}\right)}(E) .
\end{aligned}
$$

Here $\delta(E)$ is the Dirac delta function, and $\chi_{(a, b)}(E)$ is the characteristic function of the interval $(a, b)$, which is equal to 1 if $a<E<b$, and is equal to 0 if otherwise. Then $g(t)$ is expressed as

$$
g(t)=\int_{0-}^{\infty} \rho(E) \mathrm{e}^{-E t} \mathrm{~d} E .
$$




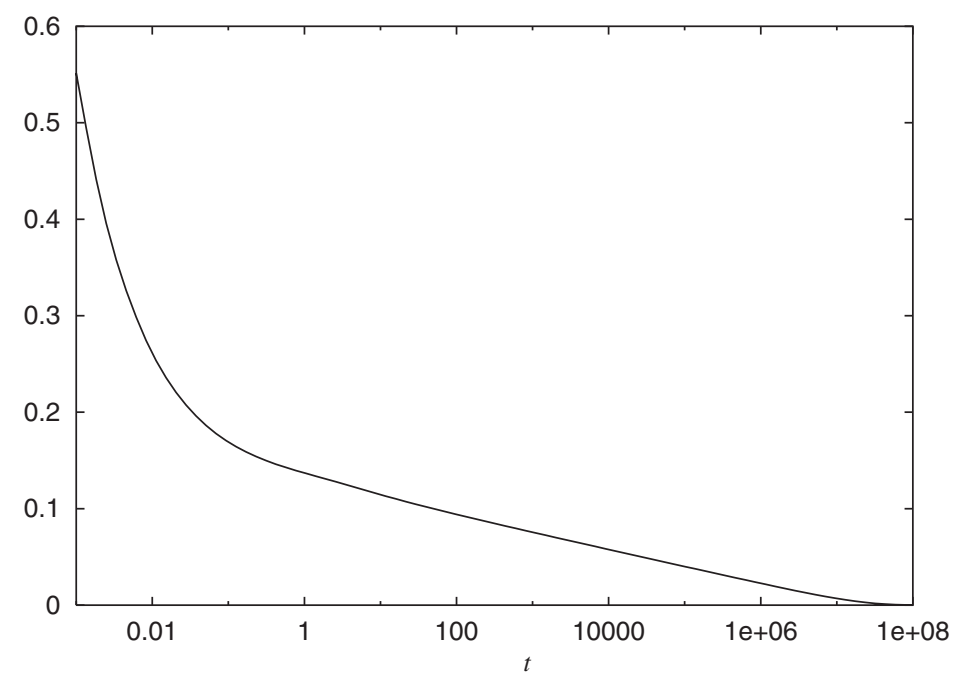

Fig. 9. Function $g(t)$ given by (27) for parameters given below (19).

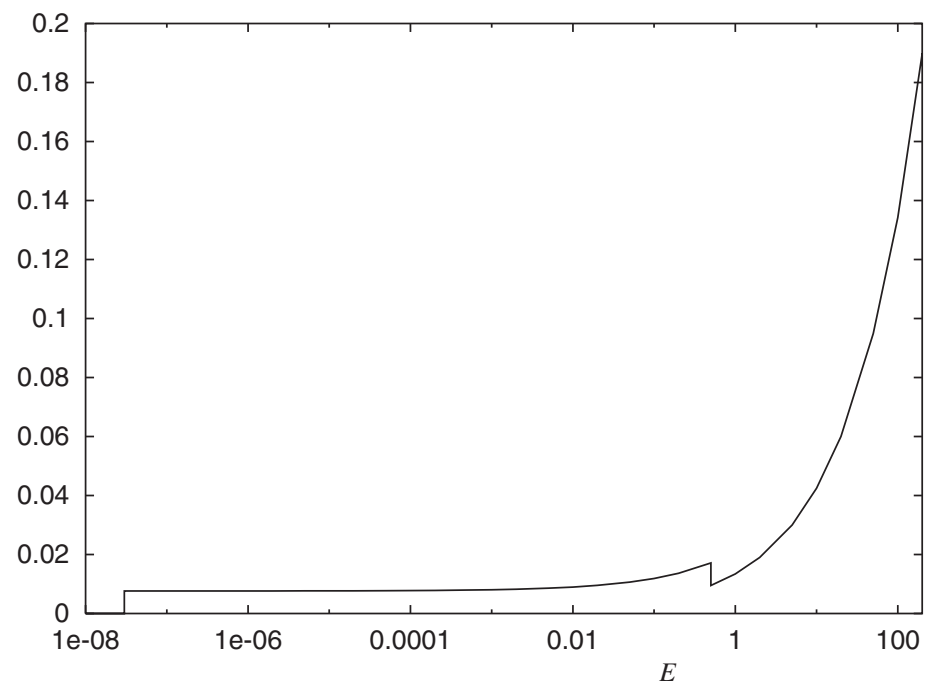

Fig. 10. $E \rho(E)$ as a function of $E$.

This shows that $g(t)$ is the Laplace transform of $\rho(E)$. Figure 10 shows the graph of $E \rho(E)$ as a function of $\lg E=\ln E / \ln 10$, since

$$
\int_{a}^{b} \rho(E) \mathrm{d} E=\int_{\ln a}^{\ln b} E \rho(E) \mathrm{d}[\ln E] .
$$

Here we observe a constant contribution between $2^{-{ }^{* *}} \omega_{0}=2^{-24} \times 0.5 \simeq 2.98 \times 10^{-8}$ and $\omega_{0}=0.5$ due to the last term in (30).

It is noted from (29) that

$$
\rho(E)=\frac{1}{\pi} \lim _{\epsilon \rightarrow 0+} \operatorname{Im}\{\hat{g}(-E-\mathrm{i} \epsilon)\},
$$

since

$$
\lim _{\epsilon \rightarrow 0+} \operatorname{Im} \frac{1}{E-\mathrm{i} \epsilon}=\pi \delta(E) .
$$

In Section 3, we have

$$
c M_{1}\left(\omega / \omega_{1}\right)=c \frac{\mathrm{i} \omega}{\mathrm{i} \omega+\omega_{1}} \frac{2}{\pi} K\left(\frac{\omega_{1}}{\mathrm{i} \omega+\omega_{1}}\right)
$$

in (10) for the contribution to $G^{*}(\omega)$. The corresponding contribution to $\hat{g}(s)$ is $c \hat{g}_{2}(s)$, if we define $\hat{g}_{2}(s)$ by 


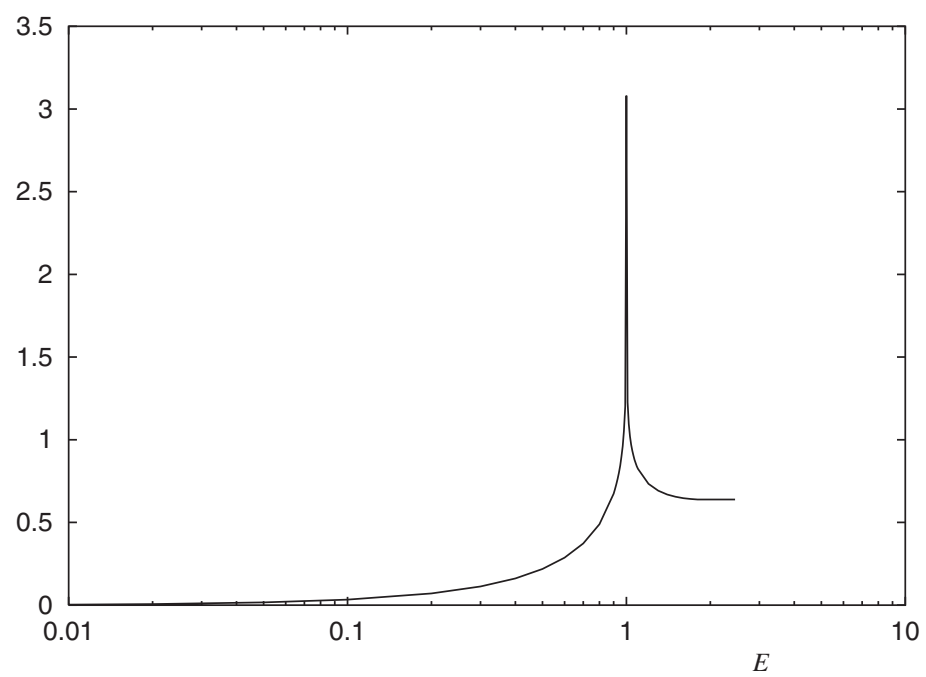

Fig. 11. $E \rho_{2}(E)$ as a function of $E$, when $\omega_{1}=1$.

$$
\hat{g}_{2}(s)=\frac{1}{s+\omega_{1}} \frac{2}{\pi} K\left(\frac{\omega_{1}}{s+\omega_{1}}\right) .
$$

The corresponding contribution to $\rho(E)$ is $c \rho_{2}(E)$ if $\rho_{2}(E)$ is calculated from $\hat{g}_{2}(s)$ by using the formula (32). Comparing (33) with (9), we see that (9) is equal to $\hat{g}_{2}(\mathrm{i} \omega)$ times $2 C_{2} \tau_{1,1} \pi^{2}$, if $\omega_{1}=4 C_{2}$. If we replace $\mathrm{i} \omega$ in (9) by $-E-\mathrm{i} \epsilon$, and calculate the imaginary part, we obtain $\rho_{2}(E)$ times $2 C_{2} \tau_{1,1} \pi^{2} \times \pi$. By using the expression in terms of the double integral in (9), we can easily confirm that $\rho_{2}(E)=0$ when $E<0$ or $E>2 \omega_{1}=8 C_{2}$, and also that

$$
\int_{-\infty}^{\infty} \rho_{2}(E) \mathrm{d} E=1
$$

Figure 11 shows the graph of $E \rho_{2}(E)$ as a function of $E$. We can regard that the range of $E$ where $E \rho_{2}(E)$ is not small, is narrow, and we can replace $\rho_{2}(E)$ by $\delta\left(E-\omega_{1}\right)$. Then $M_{1}\left(\omega / \omega_{1}\right)$ is replaced by the simplified expression $M_{0}^{*}\left(\omega / \omega_{1}\right)=\mathrm{i} \omega /\left(\mathrm{i} \omega+\omega_{1}\right)$.

Here we recall a paper by Hara et al. [4], in which the power decay of a time-dependent correlation function was derived for a system described by a set of Langevin equations. In that paper, an argument is given to discuss a power decay of $g(t)$ expressed in the form of (31) by the behaviour of $\rho(E)$. In the present case, we obtain $t^{-1 / 2}$ dependence of $g(t)$ from $E^{-1 / 2}$ dependence of $\rho(E)$, and $\ln t$ dependence of $g(t)$ from $E^{-1}$ dependence of $\rho(E)$.

\section{REFERENCES}

[1] Bagley, R. L., and Torvik, P. J., "A theoretical basis for the application of fractional calculus of viscoelasticity," J. Rheology, 27: 201-210 (1983).

[2] Feller, W., An Introduction to Probability Theory and Its Applications, 3rd ed., Vol. I, John Wiley \& Sons, New York, p. 361 (1970).

[3] Flory, P. J., Principles of Polymer Chemistry, Cornell University Press, Ithaca, NY, p. 454ff (1953).

[4] Hara, H., Lee, S. S., Koyama, J., and Fujita, S., "Scaled Langevin equation for complex systems: New linear scaling relation for weight factor," Chaos, Solitons \& Fractals, 8: 1685-1691 (1997).

[5] Magnus, W., Oberhettinger, F., and Soni, R. P., Formulas and Theorems for Special Functions of Mathematical Physics, 3rd ed., Springer-Verlag New York, New York, p. 342ff (1966).

[6] Morita, T., "Useful procedures for computing the lattice Green's function—square, tetragonal and bcc lattices," J. Math. Phys., 12: 1744-1747 (1971).

[7] Morita, T., "Calculation of the complete elliptic integrals with complex modulus," Numer. Math., 29: $233-236$ (1978).

[8] Morita, T., "Calculation of the elliptic integrals of the first and second kinds with complex modulus," Numer. Math., 82: 677688 (1999).

[9] Morita, T., "Numerical calculation of the elliptic integrals of the first and second kinds with complex modulus," Interdisciplinary Inf. Sci., 6: 67-74 (2000).

[10] Morita, T., and Horiguchi, T., "Convergence of the arithmetic-geometric mean procedure for the complex variables and the calculation of the complete elliptic integrals with complex modulus," Numer. Math., 20: 425-430 (1973).

[11] Murakami, K., Basis of Rheology (in Japanese), Sangyo-Tosho, Tokyo, p. 202ff (1991).

[12] Rouse, P. E., "A theory of the linear viscoelastic properties of dilute solutions of coiling polymers," J. Chem. Phys., 21: 12721280 (1953). 